\title{
Correlation among RKIP expression, NF- KB p65 levels, and T-lymphocyte subsets in gastric cardia adenocarcinoma
}

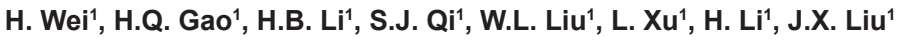 \\ and Z.M. Dong' \\ ${ }^{1}$ Hebei Engineering University Affiliated Hospital, Handan, Hebei, China \\ ${ }^{2}$ Cancer Institute, Fourth Hospital of Hebei Medical University, Hebei, China \\ Corresponding author: J.X. Liu \\ E-mail: liujianxiadr@163.com \\ Genet. Mol. Res. 14 (4): 16491-16496 (2015) \\ Received July 22, 2015 \\ Accepted October 8, 2015 \\ Published December 9, 2015 \\ DOI http://dx.doi.org/10.4238/2015.December.9.21
}

ABSTRACT. The aim of this study was to characterize variations in Raf kinase inhibitor protein (RKIP) expression and related signaling molecules in gastric cardia adenocarcinoma. Cancerous and precancerous tissues were collected from patients with gastric cardia adenocarcinoma and normal tissue was collected from healthy controls. RKIP expression was detected in these tissues and the serum levels of NF-KB p65 and T-lymphocyte subsets were measured. Positive RKIP expression was higher in gastric cardia adenocarcinoma tissues than in precancerous tissues. The serum level of total NF-KB p65 was higher in patients with gastric cardia adenocarcinoma than in healthy controls. Levels of NF-KB p65 did not correlate with positive and negative expression of RKIP, but were higher in patients with lymph node metastasis than in those without it. The cellular immune function of the gastric cardia adenocarcinoma group was lower than in normal controls, particularly in cases with negative RKIP expression. RKIP is downregulated in gastric cardia adenocarcinoma tissues, which is related to the occurrence, progression, invasion, and metastasis of tumors. The possible mechanism for this may be the inhibition 
of NF-kB activity and cellular immune function, which allows for the escape of tumor cells from immune surveillance.

Key words: Gastric cardia adenocarcinoma; T-lymphocyte subsets; Raf kinase inhibitor protein; Nuclear factor-kB p65

\section{INTRODUCTION}

The diagnosis of gastric cardia adenocarcinoma has improved because of the progress in endoscopic and pathological diagnosis techniques. Tumor occurrence is mostly related to dysregulation of signal transduction pathways. Raf kinase inhibitor protein (RKIP) is a tumor suppressor and is considered the endogenous inhibitor of the ras-mitogen-activated protein kinase (RASMAPK) signaling pathway (Al-Mulla et al., 2008; Li et al., 2008; Wang and Wang, 2008). RKIP plays an important role in tumor immunity by regulating NF-KB-mediated functions (Yeung et al., 2001). We detected expression of RKIP and serum levels of NF-KB p65 protein and T-lymphocyte subsets for 160 cases of gastric cardia adenocarcinoma. Our results have further clarified the molecularrole of RKIP in the occurrence and progression of gastric cardia adenocarcinoma.

\section{MATERIAL AND METHODS}

\section{Patient samples and clinical data}

A total of 160 gastric cardia adenocarcinoma patients receiving surgical treatment at the Affiliated Hospital of Hebei Hospital of Engineering were recruited, including 112 males and 48 females aged 37 to 83 (average $61.8 \pm 8.7$ years old; 66 cases $\leq 60$ years and 94 cases $\geq 60$ years). Primary tumors and precancerous tissues $5 \mathrm{~cm}$ away from the tumor were collected and confirmed to have no tumor cell infiltration by hematoxylin and eosin (H\&E) staining. The specimens were preserved in liquid nitrogen and fixed in $10 \%$ neutral buffered formaldehyde. According to the Union for International Cancer Control TNM staging system, 70 and 90 patients were classified as stage I + II and stage III + IV, respectively. Pathologically, 41, 54, and 65 patients were classified as highly, moderately and poorly differentiated, respectively. Additionally, 92 and 68 patients with gastric cardia adenocarcinoma presented with or without lymph node metastasis, respectively. None of the patients received preoperative chemotherapy or radiotherapy. Prior to surgical treatment, two fasting peripheral venous blood samples were collected ( $2 \mathrm{~mL}$ each time). To one sample, anticoagulant was added and peripheral blood mononuclear cells (PBMC) were harvested. For the other sample, the serum was separated and preserved at $-80^{\circ} \mathrm{C}$. Fifty healthy subjects were used as controls, including 33 males and 17 females aged 28 to 72 (average 60.2 \pm 7.7 years old).

\section{Equipment and reagents}

The following reagents, kits and equipment were used: RKIP rabbit monoclonal antibody (CapitalBio Corporation, Beijing, China); rabbit secondary antibody immunochemistry kit (Beijing Zhongshan Golden Bridge Biotechnology Co. Ltd., Beijing, China); human NF-kB p65 ELISA kit (Jingmei Biotech Co. Ltd., Beijing, China); Epics-XLII Flow Cytometer, monoclonal anti-CD3-PE antibody, monoclonal anti-CD4-FITC antibody, monoclonal anti-CD8-PE antibody, and monoclonal anti-CD25-FITC antibody (Coulter Corporation, Miami, FL, USA); and Foxp3-FITC monoclonal antibody (eBioscience, San Diego, CA, USA). 


\section{Detection of RKIP expression in gastric cardia adenocarcinoma tissues}

The standard immunochemical streptavidin-peroxidase method was employed to detect expression of RKIP. Cancerous tissues and precancerous tissues were made into paraffin sections. After conventional dewaxing, the specimens were sealed in $3 \% \mathrm{H}_{2} \mathrm{O}_{2}$ in methanol and then subjected to microwave repair for $15 \mathrm{~min}$. Primary antibody, biotinylated secondary antibody and horseradish peroxidase-labeled tertiary antibody were added successively, and color development was performed using 3,3'-diaminobenzidine (DAB) solution. The nuclei were counterstained with hematine, followed by conventional dehydration, transparentization, and sealing with neutral gum. For the negative control, PBS was added instead of primary antibody, with all other procedures being identical. The confirmed positive sections were used as the positive controls, where uniform brown granules in the cytoplasm after staining for RKIP was considered as positive. The sections were assessed independently by three experienced pathologists by referring to Fromowitz's scoring standard and the final result was based on the mean scores.

\section{Detection of serum levels of NF-KB p65 and T-lymphocyte subsets}

Serum levels of total NF-KB p65 and active NF-KB p65 were detected according to the manufacturer protocol for the ELISA kit. Prior to the detection of total NF-kB p65, $1 \mathrm{M}$ hydrochloric acid treatment was performed. Using the harvested PBMC, the ratios between CD3+, CD4+, CD8+ and CD4+-CD25+-Foxp3+ circulating regulatory T cells (Treg) were detected by flow cytometry.

\section{Statistical analysis}

SPSS11.5 software was used for statistical analysis. Data are expressed as mean \pm standard deviation, and inter-group comparisons were carried out using the $t$-test. Data where counting was performed are expressed as relative numbers. The relationship between two variables was measured by Spearman's rank correlation coefficient and correlation analysis, and P $\leq 0.05$ was considered statistically significant.

\section{RESULTS}

\section{RKIP expression in cancerous and precancerous tissues}

Positive RKIP expression in cancerous and precancerous tissues was 38.7\% (62/160 patients) and $52.5 \%$ (84/160 patients), respectively $(P<0.05)$. RKIP expression in cancerous tissues was independent of the patient's age, gender, TNM staging, and tumor differentiation degree $(\mathrm{P}>$ 0.05). Positive RKIP expression in those patients with or without lymph node metastasis was 24.4 and $53.1 \%$, respectively $(P<0.05, t=4.34)$.

\section{Serum level of NF-KB p65}

The serum level of total NF-kB p65 in the gastric cardia adenocarcinoma group was higher than the control group $(P<0.05)$. However, this did not correlate with positive or negative RKIP expression $(P>0.05)$. However, patients with lymph node metastasis had a higher level of total NF-kB p65 than patients without lymph node metastasis $(P<0.05)$. These results are summarized in Table 1. 
Table 1. Comparison of serum levels of NF-KB p65 between groups ( $\mu \mathrm{g} / \mathrm{L}$, means $\pm \mathrm{SD})$.

\begin{tabular}{lcc}
\hline Group & Total NF-KB p65 & Active NF-kB p65 \\
\hline Gastric cardia adenocarcinoma & $18.3 \pm 5.5$ & $8.3 \pm 5.0$ \\
Negative RKIP expression & $18.0 \pm 4.0$ & $7.9 \pm 3.0$ \\
Positive RKIP expression & $18.3 \pm 5.1$ & $8.5 \pm 4.2$ \\
Without lymph node metastasis & $14.0 \pm 4.7$ & $8.3 \pm 3.0$ \\
With lymph node metastasis & $21.7 \pm 5.0$ & $8.1 \pm 4.2$ \\
Control & $11.2 \pm 3.0$ & $8.7 \pm 4.2$ \\
\hline
\end{tabular}

\section{Serum levels of T-lymphocyte subsets}

The CD3+ and CD4+ cell counts and CD4+/CD8+ ratios in the gastric cardia adenocarcinoma group were all lower than the control group, while the CD8+ and Treg cell counts were higher in the gastric cardia adenocarcinoma group than the control group $(P<0.05)$. The CD3+ and CD4+ cell counts and CD4+/CD8+ ratio in patients with lymph node metastasis were lower than in patients without lymph node metastasis, and CD8+ and Treg cell counts were higher in patients with or without lymph node metastasis compared to the control group $(P<0.05)$. The CD4+ cell count and CD4+/CD8+ ratio were higher in patients with positive RKIP expression than those with negative RKIP expression, while CD8+ and Treg cell counts of patients with positive RKIP expression were lower than in patients with negative RKIP expression $(P<0.05)$. These results are summarized in Table 2.

Table 2. Comparison of serum levels of T-lymphocyte subsets between groups (means $\pm S D$ ).

\begin{tabular}{|c|c|c|c|c|c|}
\hline Group & $\mathrm{CD} 3+(\%)$ & $\mathrm{CD} 4+(\%)$ & $\mathrm{CD} 8+(\%)$ & CD4+/CD8+ & Treg $(\%)$ \\
\hline Gastric cardia adenocarcinoma & $53.4 \pm 6.5$ & $31.9 \pm 5.6$ & $36.1 \pm 4.9$ & $0.7 \pm 0.1$ & $7.0 \pm 1.6$ \\
\hline Without lymph node metastasis & $67.0 \pm 5.7$ & $39.2 \pm 4.9$ & $28.5 \pm 5.3$ & $1.0 \pm 0.2$ & $5.7 \pm 1.0$ \\
\hline With lymph node metastasis & $38.1 \pm 4.8$ & $25.1 \pm 5.8$ & $40.1 \pm 5.9$ & $0.4 \pm 0.1$ & $8.0 \pm 1.8$ \\
\hline Negative RKIP expression & $52.6 \pm 4.9$ & $26.2 \pm 6.1$ & $39.6 \pm 3.9$ & $0.6 \pm 0.1$ & $7.7 \pm 1.3$ \\
\hline Positive RKIP expression & $53.7 \pm 5.6$ & $37.1 \pm 4.9$ & $29.9 \pm 4.8$ & $0.9 \pm 0.2$ & $6.0 \pm 0.9$ \\
\hline Control & $65.5 \pm 5.5$ & $43.6 \pm 4.9$ & $25.6 \pm 6.1$ & $1.4 \pm 0.3$ & $5.0 \pm 0.6$ \\
\hline
\end{tabular}

\section{DISCUSSION}

Gastric cardia adenocarcinoma is now considered a distinct type of gastric cancer but is often diagnosed as esophageal cancer due to similar epidemiological and clinical manifestations. Progress in endoscopic screening and pathological diagnosis techniques have made it possible to establish gastric cardia adenocarcinoma as an independent disease. Widely distributed in human tissues, RKIP acts as a tumor suppressor (Fu et al., 2003; Schuierer et al., 2004; Hagan et al., 2005; Fu et al., 2006), and adenocarcinoma cases with downregulation or deletion of the RKIP gene are predicted to have a poor prognosis. We found that the incidence of positive expression of RKIP in gastric cardia adenocarcinoma was significantly lower than in precancerous tissues, particularly in those patients with lymph node metastasis. This finding further implicates RKIP in the progression, invasion, and metastasis of gastric cardia adenocarcinoma (Wei et al., 2014). In mammals, NF-KB binds specifically to promoter and enhancer sequences in a variety of cells. Through its regulatory action on many important cell factors, adhesion molecules, chemotactic factors, growth factors and immune receptors, NF-KB plays an active role in the immune response, inflammatory response and cell growth regulation (Karin and Greten, 2005). NF-kB/Rel family members 
form NF-kB homo- and heterodimers. In mammals, the NF-kB/Rel family is comprised of NF-kB1 (p50/p105), NF-kB2 (p52/p100), Rel A (p65), Rel B, and c-Rel (Yeung et al., 2010). Eves et al. (2006) found that inhibition of RKIP expression enhanced NF-kB-mediated transcription. Studies have shown that NF-KB p65 is expressed in a variety of tumor tissues, but its content in peripheral blood is rarely investigated. We found that the serum level of total NF-KB p65 in gastric cardia adenocarcinoma cases was significantly higher than control, and was also higher in patients with lymph node metastasis than in patients without lymph node metastasis. It can be inferred that the serum level of total NF-KB p65 is related to the occurrence, progression, invasion, and metastasis of gastric cardia adenocarcinoma. Moreover, the level of active NF-kB p65 inpatients with negative RKIP expression was only slightly lower than in patients with positive RKIP expression. The possible reason for this may be that NF-KB activation is controlled by various factors and the inhibitor protein of NF-KB (IXB) is a negative regulator of NF-KB p65 activation (Kearns et al., 2006). Therefore, low expression of RKIP may only have a small effect on the activation of NF-KB p65, which is not manifested in peripheral blood.

Tumor immunity mainly occurs on the level of cellular immunity, with T-lymphocyte subsets playing a crucial role in anti-cancer surveillance. Exhibiting a regulatory effect on cellular immunity, NF-kB enhances the host defense function of macrophages and neutrophil granulocytes and participates in antigen presentation in dendritic cells and T cell activation. Inhibition of NF-kB activity and knockout of NF-kB-dependent genes can lead to host immune dysfunction and impaired virus clearance ability (Sadikot et al., 2006). Animal experiments indicate that p50 (NF-kB1)-deficient mice not only present with a decline in virus clearance ability, but also an increased susceptibility to Streptococcus pneumoniae infection. Typically, the immune system maintains a relatively stable state with T-lymphocytes and Treg cells. Mouse antibodies classified as cluster of differentiation (CD) can recognize the antigenic determinant on the surface of T lymphocytes. CD3 is the surface marker of mature T cells, while CD4 and CD8 are the surface markers of T helper cells and cytotoxic T cells, respectively. A decline in CD3 cell count and abnormal CD4/CD8 ratios is usually indicative of impaired cellular immunity. As a surface marker of Treg cells, CD25, when highly expressed, has an inhibitory effect on innate immunity. Foxp3 is widely recognized as a marker of CD4+Treg cells. We found that CD3+ and CD4+ cell counts and CD4+/CD8+ ratios in gastric cardia adenocarcinoma cases were significantly lower thanin the control, while CD8+ and Treg cell counts of gastric cardia adenocarcinoma were higher than in the control. The variations in the antigens described above were also related to lymph node metastasis and RKIP expression. We infer that downregulation or deletion of the RKIP gene may affect the NF-KB-mediated positive feedback mechanism and lead to the activation of upstream regulators. This will cause the inhibition of NF-KB activity (Papa et al., 2004) and the decline of cellular immunity. Furthermore, extracellular lipopolysaccharides and tumor necrosis factor- $\alpha$ (TNF- $\alpha$ ) can also inhibit NF-KB by stimulating the production of antiinflammatory cytokines such as interleukin-10 and -B.

In conclusion, RKIP expression is closely associated with the occurrence, progression, invasion, and metastasis of gastric cardia adenocarcinoma tumors. The possible mechanism for this is the inhibition of NF-KB activity and cellular immunity, which makes it possible for tumor cells to evade immune surveillance.

\section{Conflicts of interest}

The authors declare no conflict of interest. 


\section{REFERENCES}

Al-Mulla F, Hagan S, Al-Ali W, Jacob SP, et al. (2008). Raf kinase inhibitor protein: mechanism of loss of expression and association with genomic instability. J. Clin. Pathol. 61: 524-529.

Eves EM, Shapiro P, Naik K, Klein UR, et al. (2006). Raf kinase inhibitory protein regulates aurora B kinase and the spindle checkpoint. Mol. Cell 23: 561-574.

Fu Z, Smith PC, Zhang L, Rubin MA, et al. (2003). Effects of raf kinase inhibitor protein expression on suppression of prostate cancer metastasis. J. Natl. Cancer Inst. 95: 878-889.

Fu Z, Kitagawa Y, Shen R, Shah R, et al. (2006). Metastasis suppressor gene Raf kinase inhibitor protein (RKIP) is a novel prognostic marker in prostate cancer. Prostate 66: 248-256.

Hagan S, Al-Mulla F, Mallon E, Oien K, et al. (2005). Reduction of Raf-1 kinase inhibitor protein expression correlates with breast cancer metastasis. Clin. Cancer Res. 11: 7392-7397.

Karin M and Greten FR (2005). NF-kappaB: linking inflammation and immunity to cancer development and progression. Nat. Rev. Immunol. 5: 749-759.

Kearns JD, Basak S, Wemer SL, Huang CS, et al. (2006). IkappaB epsilon provides negative feedback to control NF-kappaB oscillations, signaling, dynamics, and inflammatory gene expression. J. Cell Biol. 173: 659-664.

$\mathrm{Li} \mathrm{H}$, Wu KJ, Li S and Chen YX (2008). Regulation role of Raf kinase inhibitory protein to mitogen-activated protein kinase signaling pathway. GuojiKouqiangYixueZazhi 35: 311-312.

Papa S, Zazzeroni F, Bubici C, Jayawardena S, et al. (2004). Gadd45 beta mediates the NF-kappaB suppression of JNK signaling by targeting MKK7/JNKK2. Nat. Cell Biol. 6: 146-153.

Schuierer MM, Bataille F, Hagan S, Kolch W, et al. (2004). Reduction in Raf kinase inhibitor protein expression is associated with increased Ras-extracellular signal-regulated kinase signaling in melanoma cell lines. Cancer Res. 64: 5186-5192.

Sadikot RT, Zeng H, Joo M, Everhart MB, et al. (2006). Targeted immunomodulation of the NF-kappaB pathway in airway epithelium impacts host defense against Pseudomonas aeruginosa. J. Immunol. 176: 4923-4930.

Wang J and Wang D (2008). Raf kinase inhibitor protein (PKIP) and tumor. Sichuan Yixue 29: 469.

Wei H, Qi SJ, Liu WL, Xu L, et al. (2014). Expression changes of Raf kinase inhibitor protein and E-cadherin in gastric cardia adenocarcinoma and their correlation. Shandong Yiyao 54: 23-25.

Yeung KC, Rose DW, Dhillon AS, Yaros D, et al. (2001). Raf kinase inhibitor protein interacts with NF-kB-inducing-kinase and TAK1 and inhibits NF-kB activation. Mol. Cell Biol. 21: 7207-7217. 